\title{
Erratum to: Neuropathological assessments of the pathology in frontotemporal lobar degeneration with TDP43-positive inclusions: an inter-laboratory study by the BrainNet Europe consortium
}

\author{
Irina Alafuzoff • Maria Pikkarainen - Manuela Neumann • Thomas Arzberger • Safa Al-Sarraj • \\ Istvan Bodi • Nenad Bogdanovic • Orso Bugiani · Isidro Ferrer • Ellen Gelpi • Stephen Gentleman • \\ Giorgio Giaccone • Manuel B. Graeber • Tibor Hortobagyi • Paul G. Ince • James W. Ironside • \\ Nikolaos Kavantzas • Andrew King • Penelope Korkolopoulou • Gábor G. Kovács • David Meyronet • \\ Camelia Monoranu • Tatjana Nilsson • Piero Parchi • Efstratios Patsouris • Tamas Revesz • \\ Wolfgang Roggendorf • Annemieke Rozemuller • Danielle Seilhean • Nathalie Streichenberger • \\ Dietmar R. Thal - Stephen B. Wharton - Hans Kretzschmar
}

Published online: 25 November 2014

(C) Springer-Verlag Wien 2014

\section{Erratum to: J Neural Transm DOI 10.1007/s00702-014-1304-1}

The original version of this article unfortunately contained a mistake. The affiliation of Dr. Piero Parchi is incorrect. The correct affiliation should be:

The online version of the original article can be found under doi:10.1007/s00702-014-1304-1.

\section{Alafuzoff $(\square)$}

Section of Clinical Pathology Uppsala University Hospital, Rudbeck's Laboratory, Department of Immunology, Genetics and Pathology, Uppsala University, 75185 Uppsala, Sweden e-mail: irina.alafuzoff@igp.uu.se

\section{Pikkarainen}

Department of Clinical Medicine, University of Eastern Finland, Kuopio, Finland

\section{Neumann}

Department of Neuropatology, German Center of

Neurodegenerative Diseases, University of Tubingen and DZNE,

Tubingen, Germany

\section{T. Arzberger $\cdot$ H. Kretzschmar}

Centre for Neuropathology and Prion Research, München

Ludwig-Maximilians-University, Munich, Germany

\section{S. Al-Sarraj · I. Bodi · A. King}

Department of Clinical Neuropathology, Institute of Psychiatry,

King's College Hospital and MRC London Neurodegenerative

Diseases Brain Bank, London, UK

N. Bogdanovic

Geriatric Department, Institute for Clinical Medicine, Oslo

University, Oslo, Norway

\section{P. Parchi}

Department of Biomedical and Neuromotor Sciences, University of Bologna and IRCCS Istituto delle Scienze Neurologiche, Bologna, Italy

O. Bugiani · G. Giaccone

Division of Neuropathology and Neurology 5, IRCSS

Foundation Istituto Neurologico Carlo Besta, Milan, Italy

I. Ferrer

Institute of Neuropathology, Bellvitge University Hospital,

University of Barcelona, CEBERNED, Barcelona, Spain

E. Gelpi

Neurological Tissue Bank of the Biobanc-Hospital Clínic-

IDIBAPS, Barcelona, Spain

S. Gentleman

Neuropathology Unit, Department of Medicine, Imperial College London, London, UK

M. B. Graeber

Faculty of Medicine and Faculty of Health Science, Brain and

Mind Research Institute, The University of Sydney, Sydney,

Australia

T. Hortobagyi

Department of Neuropathology, Institute of Pathology,

University of Debrecen, Debrecen, Hungary 
P. G. Ince - S. B. Wharton

Sheffield Institute for Translational Neuroscience, University of Sheffield, Sheffield, UK

J. W. Ironside

National CJD Research \& Surveillance Unit, Western General Hospital, University of Edinburgh, Edinburgh, UK

N. Kavantzas · P. Korkolopoulou · E. Patsouris Department of Pathology, National and Capodistrian University of Athens, Athens, Greece

\section{G. G. Kovács}

Institute of Neurology, Medical University of Vienna, Vienna, Austria

D. Meyronet $\cdot$ N. Streichenberger

Hospices Civils de Lyon, Centre de Pathologie et de Neuropathologie Est, Lyon Neuroscience Research Center, Universite Lyon, Lyon, France

C. Monoranu - W. Roggendorf

Abteilung Neuropathologie, Pathologisches Institut der

Univeristät Wurzburg, Wurzburg, Germany

T. Nilsson

Department of Geriatrics, Karolinska Institutet, Huddinge, Stockholm, Sweden
P. Parchi

Department of Biomedical and Neuromotor Sciences, University of Bologna and IRCCS Istituto delle Scienze Neurologiche, Bologna, Italy

\section{T. Revesz}

Department of Molecular Neuroscience, Queen Square Brain Bank, UCL Institute of Neurology, London, UK

\section{A. Rozemuller}

VU University Medical Center, Amsterdam, The Netherlands

D. Seilhean

Laboratoire de Neuropathologie Raymond Escourolle, Assistance Publique-hopitaux de Paris, Université Pierre et Marie Curie and INSERM, Paris, France

\section{R. Thal}

Institute of Pathology, Laboratory of Neuropathology, University of Ulm, Ulm, Germany 